\title{
Evaluation of immune responses and analysis of the effect of vaccination of the Leishmania major recombinant ribosomal proteins L3 or L5 in two different murine models of cutaneous leishmaniasis
}

\author{
Laura Ramírez $^{\mathrm{a}, 1}$, Diego M. Santos ${ }^{\mathrm{b}, 1}$, Ana P. Souza ${ }^{\mathrm{b}}$, Eduardo A.F. Coelho ${ }^{\mathrm{c}}$, Aldina Barral ${ }^{\mathrm{b}}$, \\ Carlos Alonso $^{a}$, Marta R. Escutia $^{\mathrm{d}}$, Pedro Bonay ${ }^{\mathrm{a}}$, Camila I. de Oliveira $^{\mathrm{b}, 1}$, Manuel Soto ${ }^{\mathrm{a}, *, 1}$ \\ a Centro de Biología Molecular Severo Ochoa (CSIC-UAM), Departamento de Biología Molecular, Nicolás Cabrera 1, Universidad Autónoma de Madrid, 28049 Madrid, Spain \\ b Centro de Pesquisas Gonçalo Moniz (Fundação Oswaldo Cruz- FIOCRUZ) 40.296-710 Salvador, Bahia, Brazil

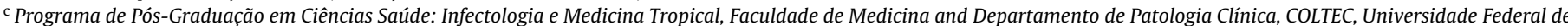 \\ Minas Gerais, 31.270-901, Belo Horizonte, Minas Gerais, Brazil \\ d Research E Development Department, Laboratorios LETI S.L.u., 28760 Madrid, Spain
}

\section{A R T I C L E I N F O}

\section{Article history:}

Received 21 August 2012

Received in revised form

11 December 2012

Accepted 24 December 2012

Available online 10 January 2013

\section{Keywords:}

Leishmania

BALB/c mice

Th1/Th2 Immune responses

Recombinant ribosomal proteins

Vaccines

\begin{abstract}
A B S T R A C T
Four new antigenic proteins located in Leishmania ribosomes have been characterized: S4, S6, L3 and L5. Recombinant versions of the four ribosomal proteins from Leishmania major were recognized by sera from human and canine patients suffering different clinical forms of leishmaniasis. The prophylactic properties of these proteins were first studied in the experimental model of cutaneous leishmaniasis caused by L. major inoculation into BALB/C mice. The administration of two of them, LmL3 or LmL5 combined with $\mathrm{CpG}$-oligodeoxynucleotides (CpG-ODN) was able to protect BALB/c mice against $L$. major infection. Vaccinated mice showed smaller lesions and parasite burden compared to mice inoculated with vaccine diluent or vaccine adjuvant. Protection was correlated with an antigen-specific increased production of IFN- $\gamma$ paralleled by a decrease of the antigen-specific IL-10 mediated response in protected mice relative to non-protected controls. Further, it was demonstrated that BALB/c mice vaccinated with recombinant LmL3 or LmL5 plus CPG-ODN were also protected against the development of cutaneous lesions following inoculation of $L$. braziliensis. Together, data presented here indicate that LmL3 or LmL5 ribosomal proteins combined with Th1 inducing adjuvants, may be relevant components of a vaccine against cutaneous leishmaniasis caused by distinct species.
\end{abstract}

(C) 2013 Elsevier Ltd. All rights reserved.

\section{Introduction}

Infection with Leishmania protozoan parasites can result in the development of leishmaniasis. Several species cause cutaneous leishmaniasis (CL) including Leishmania major and L. braziliensis (in the Old or New World, respectively); the latter is also related with development of mucocutaneous leishmaniasis (MCL). Visceral leishmaniasis (VL) is caused by the infection of L. chagasi in the New World or L. infantum and L. donovani in the Old World [1]. In natural and experimental leishmaniasis, effective primary immunity requires the production of IFN- $\gamma$ by $C D 4^{+} \mathrm{T}$ cells and to a minor

Abbreviations: CpG-ODN, CpG-oligodeoxynucleotides; VL, visceral leishmaniasis; MCL, mucocutaneous leishmaniasis; CL, cutaneous leishmaniasis; LRP, Leishmania ribosomal proteins; SLA, soluble Leishmania antigens; MRP, mouse ribosomal proteins; CR, coding region; DLN, draining lymph nodes; OPD, ortophenylediaminedihydrochloride.

* Corresponding author. Tel.: +34 91196 4471; fax: +34 911964420

E-mail address: msoto@cbm.uam.es (M. Soto).

1 These authors contributed equally to this work. extend by $\mathrm{CD}^{+} \mathrm{T}$ cells, which mediates nitric-oxide-depending parasite killing by the activation of infected macrophages [2]. Disease progression is related with the induction of humoral and IL-10 mediated responses [3].

A preparation of biochemically purified Leishmania ribosomal proteins (LRP) administered with CpG-oligodeoxynucleotides (CpG-ODN) conferred protection against challenge with $L$. major parasites in susceptible BALB/c and resistant C57BL/6 mice. Protection was correlated with a LRP-specific IL-12 dependent production of IFN- $\gamma$ (in both mouse strains) and a diminished production of both IL-4 and IL-10 in BALB/c mice [4]. Also, BALB/c mice vaccinated with LRP plus CpG-ODN and subsequently infected were able to resist a secondary challenge [5]. A protective response against $L$. chagasi and $L$. amazonensis was observed in BALB/C mice when a LRP-specific IFN- $\gamma$ mediated response was induced by administration of LRP combined with saponin [6].

In this work, four L. major ribosomal antigens have been characterized: LmS4, LmS6, LmL3 and LmL5. Their prophylactic properties were assayed in the $L$. major-BALB/c mouse model of CL. Since immunization of LmL3 or LmL5 combined with CpG-ODN elicited 
A
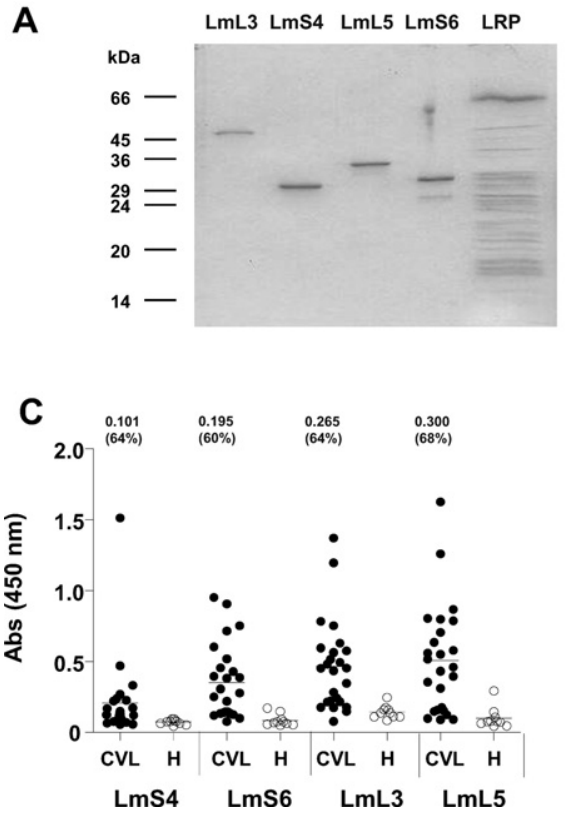

B
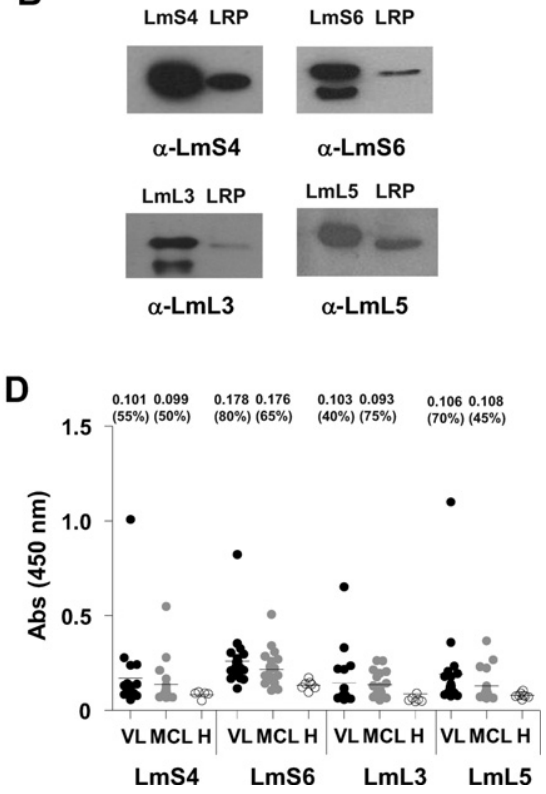

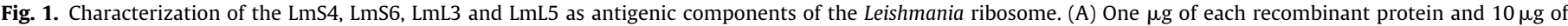

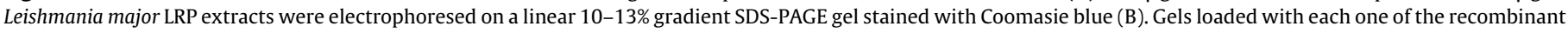

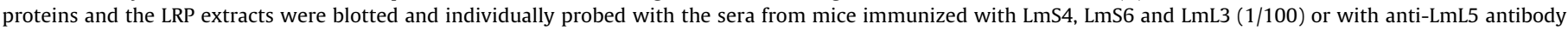

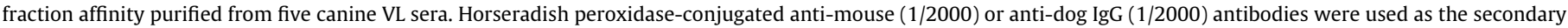

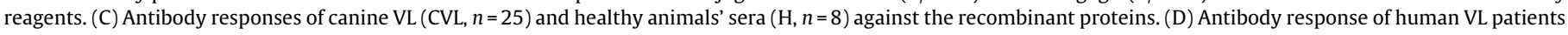

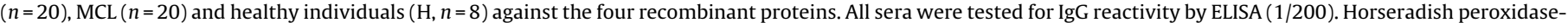

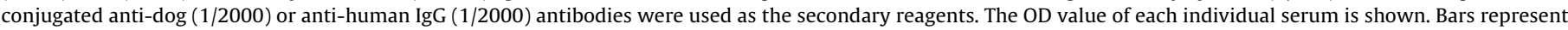

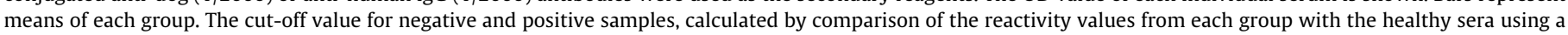

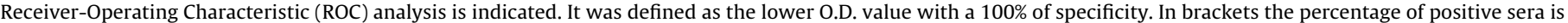
also indicated.

protective responses against $L$. major infection, their capacity to modulate L. braziliensis infection in the same mice strain was tested. Mice vaccinated with both proteins were able to control parasite growth in the site of infection in this New World species experimental model.

\section{Materials and methods}

\subsection{Mice strains and parasites}

Female BALB/c mice (6-8 week old) were purchased from Harlan (BCN, Spain) or were obtained from the Centro de Pesquisa Gonçalo Moniz, FIOCRUZ. Promastigotes of $L$. major strains (WHOM/IR/-/173) or clone V1 (MHOM/IL/80/Friedlin) and from L. braziliensis (MHOM/BR/01/BA788), were cultured at $26^{\circ} \mathrm{C}$ in Schneider medium (Gibco, NY) supplemented with $10 \%$ fetal calf serum, $100 \mathrm{U} / \mathrm{ml}$ of penicillin and $100 \mu \mathrm{g} / \mathrm{ml}$ of streptomycin.

\section{2. $C p G-O D N$}

Phosphorothioate-modified CPG-ODN (5'-TCAACGTTGA-3' and 5'-GCTAGACGTTAGCGT-3') were synthesized by Isogen (The Netherlands) and employed for their capacity to induce Th1 responses in mice when immunized with various leishmanial antigenic preparations $[7,8]$.

\subsection{Cloning of DNA sequences coding for L. major ribosomal proteins LmS4, LmS6, LmL3 and LmL5}

The L. major LmS4, LmS6, LmL3 and LmL5 coding regions were obtained from the $L$. major genome database (www.genedb.org/genedb/leish) using the Saccharomyces cerevisiae orthologous protein sequences as probes [9]. Coding regions were PCR amplified using specific primers (Supplementary Fig. 1) and the DNA from L. major (MHOM/IL/80/Friedlin). Amplified DNAs were cloned into the pQE30 prokaryotic expression vector (Qiagen, Germany). The four clones were double-stranded sequenced in the same plasmid.

\subsection{Protein purification}

Recombinant proteins were over-expressed in Escherichia coli, purified under denaturing conditions onto $\mathrm{Ni}$-nitrilotriacetic-acidagarose columns (Qiagen) and refolded on the affinity column as described [10]. Polymyxin-agarose columns (Sigma, MO) were employed to remove residual endotoxin content $(<10 \mathrm{pg}$ of LPS per $1 \mu \mathrm{g}$ of recombinant protein, measured by the Quantitative Chromogenic Limulus Amebocyte Assay QCL-1000 (BioWhittaker, MD)).

\subsection{Sera, immunoblotting and ELISA assays}

Mice sera were collected at the beginning of the experiment, before challenge with parasites and at the 7 th week after challenge with L. major. Human VL and MCL sera were obtained from clinical and parasitologically diagnosed Brazilian patients. Canine symptomatic VL sera were collected in the Extremadura region of Spain [11]. Control sera were obtained from healthy individuals.

Soluble Leishmania antigens (SLA), mouse ribosomal proteins (MRP) and LRP were prepared as described in [4]. For immunoblotting, recombinant proteins and LRP extracts were electrophoresed and blotted as described in [11]. Anti-LmL3, anti-LmS4 or anti-LmS6 polyclonal sera were obtained from the immunized mice described below. Anti-LmL5 antibodies were obtained by passing canine VL sera through a recombinant LmL5 affinity chromatography column prepared as in [12]. For ELISA, recombinant proteins were used at $1.0 \mu \mathrm{g}$ per well. Murine, canine and human sera were employed 
A

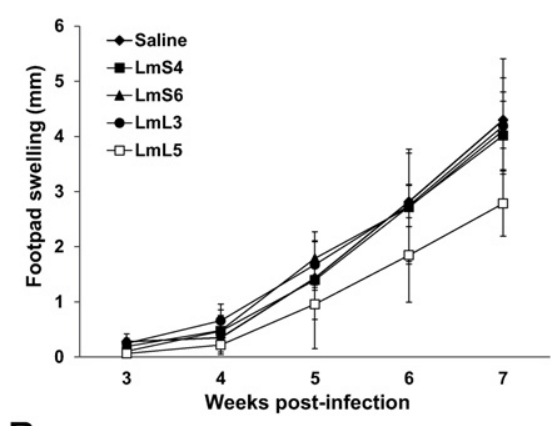

B

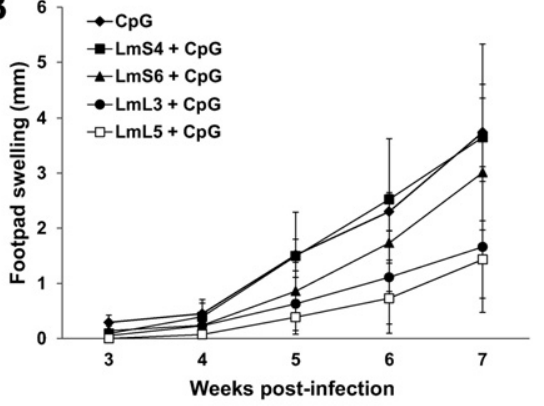

\begin{tabular}{|l|rcc|}
\hline Group Week & $5^{\text {th }}$ & $6^{\text {th }}$ & $7^{\text {th }}$ \\
\hline LmS4 & 0.91 & 0.88 & 0.45 \\
LmS6 & 0.83 & 0.83 & 0.77 \\
LmL3 & 0.28 & 0.75 & 0.82 \\
LmL5 & 0.41 & 0.18 & 0.10 \\
\hline
\end{tabular}

D

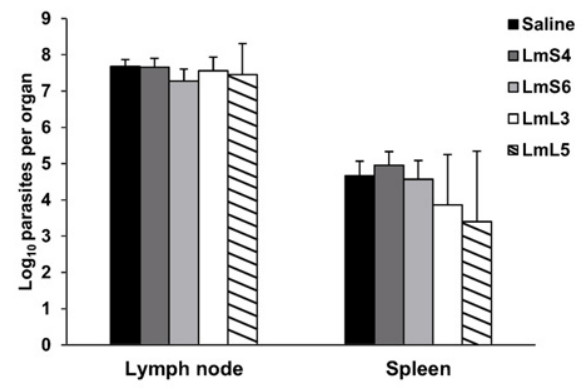

E

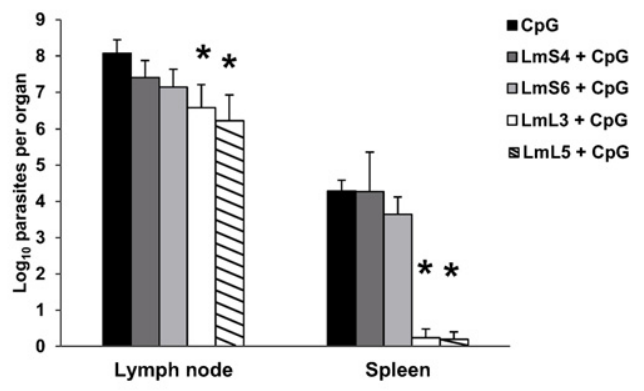

C

\begin{tabular}{|l|lll|}
\hline Group Week & $5^{\text {th }}$ & $6^{\text {th }}$ & $7^{\text {th }}$ \\
\hline LmS4 + CpG & 0.68 & 0.96 & 0.91 \\
LmS6 + CpG & 0.05 & 0.23 & 0.38 \\
LmL3 + CpG & 0.01 & 0.05 & 0.02 \\
LmL5 + CpG & 0.001 & 0.01 & 0.03 \\
\hline
\end{tabular}

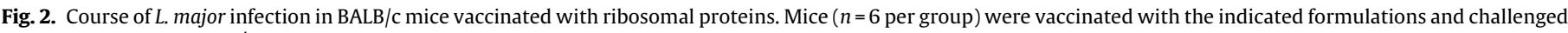

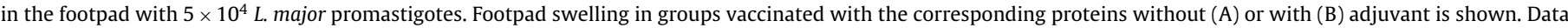

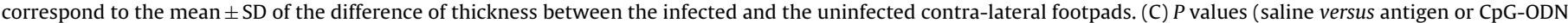

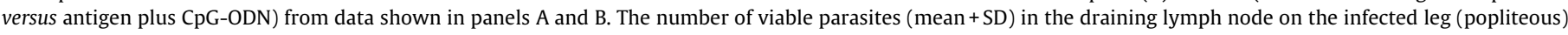

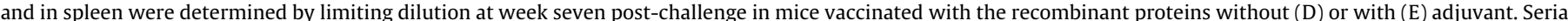

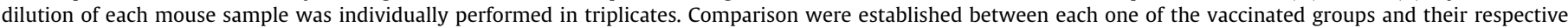

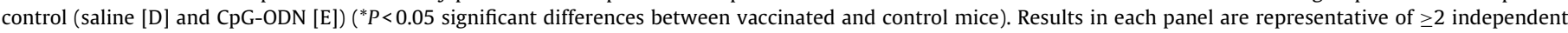
experiments.

as described in $[4,12,13]$, respectively. Secondary antibodies were purchased from Nordic (Tilburg, The Netherlands). The reciprocal endpoint titre, defined as the inverse of the highest serum dilution factor giving an absorbance $>0.15$ was determined by serial dilution of the sera.

\subsection{Immunizations, parasite challenge and parasite quantification}

For the L. major-BALB/c model, mice ( $n=6$ per group) were independently inoculated in the right hind footpad with $10 \mu \mathrm{g}$ of each recombinant protein (LmS4, LmS6, LmL3 or LmL5), alone or combined with $25 \mu \mathrm{g}$ of each CpG-ODN. As control groups, mice were inoculated with $25 \mu \mathrm{g}$ of each CpG-ODN or with saline. Each group was boosted two and four weeks later with the same dose. Parasite challenge was carried out by subcutaneous inoculation with $5 \times 10^{4}$ stationary-phase promastigotes of $L$. major (WHOM/IR//173) into the left footpad, four weeks after the last immunization. Footpad swelling was measured with a metric caliper (thickness of the left footpad minus thickness of the right footpad). For the L. braziliensis model BALB/c mice ( $n=5$ per group) were intradermically (i.d.) inoculated with $10 \mu \mathrm{g}$ of each recombinant protein (LmL3 or LmL5) combined with $25 \mu \mathrm{g}$ of each CpG-ODN or with a mixture of the proteins ( $5 \mu \mathrm{g}$ each) plus $25 \mu \mathrm{g}$ of each CpG-ODN in the left ear. Inoculation schedule was the same indicated above. Mice were challenged one month after the last inoculation in the dermis of the right ear with $1 \times 10^{5}$ stationary-phase promastigotes of $L$. braziliensis in the presence of sand fly saliva, as described [14]. Ear thickness was monitored weekly using a caliper. For parasite load determination the ears (in the case of L. braziliensis infection), draining lymph nodes (DLN) and spleen from each mouse were independently processed as described in Ref. [7] and assayed in triplicates by limiting dilution [15].

\subsection{Measurement of cytokines}

The release of IFN- $\gamma$, IL-10 and IL- 4 was measured in culture supernatants of splenocytes or DLN cells obtained from the different mice groups, following stimulation with the corresponding recombinant proteins, using commercial ELISA kits (eBioscience, CA) as described [4]. Briefly, spleen or lymph node cells obtained from each mouse were seeded and independently cultured (at $5 \times 10^{6}$ cells per $\mathrm{ml}$ ) during $48 \mathrm{~h}$ at $37^{\circ} \mathrm{C}$ alone or with the next stimuli: recombinant $\mathrm{LmL3}(10 \mu \mathrm{g} / \mathrm{ml})$, recombinant LmL5 $(10 \mu \mathrm{g} / \mathrm{ml})$, SLA $(12 \mu \mathrm{g} / \mathrm{ml})$ or MRP $(12 \mu \mathrm{g} / \mathrm{ml})$. When indicated cells were stimulated with a mixture of the two recombinant proteins ( $5 \mu \mathrm{g} / \mathrm{ml}$ each one).

\subsection{Statistical analysis}

The Receiver Operating Characteristic (ROC) curves were used to analyze the data obtained with sera samples from patients. Statistical analysis with the vaccinated and infected mice was performed 
by a two-tailed Student's $t$-test. Differences were considered significant when $P<0.05$.

\section{Results}

\subsection{Leishmania ribosomal proteins $S 4, S 6, L 3$ and $L 5$ are} antigenic in canine and human leishmaniasis

The putative L. major S4, S6, L3 and L5 ribosomal proteins were identified using as probes the $S$. cerevisiae homologous aminoacid sequences [9] in a BLASTP search (Supplementary Fig. 2A). The degree of sequence identity with yeast and human ribosomal proteins (Supplementary Fig. 2A-B) supports the statement that they are components of ribosomes. In addition, antibodies specific for each protein revealed single bands with the expected molecular weights in a LRP preparation by Western blot (Fig. 1A and B). A high degree of sequence identity was observed for the proteins in different Leishmania species (Supplementary Fig. 2B).

The four recombinant proteins were recognized by the sera from dogs affected by VL (Fig. 1C). The percentages of positive sera ranged from 60 to $68 \%$ (Fig. 1C). They were also recognized by sera samples from Brazilian patients with VL and MCL, infected by L. chagasi and L. braziliensis, respectively (Fig. 1D).

\subsection{Outcome of $C L$ due to $L$. major following vaccination of $B A L B / c$ mice with recombinant ribosomal proteins}

Next, we analyzed whether the immunization with the respective recombinant proteins was able to induce protection against L. major infection. Different groups of BALB/c mice were independently vaccinated with each recombinant protein in the absence or in the presence of $\mathrm{CpG}-\mathrm{ODN}$. Mice groups inoculated with the vaccine diluent (saline) or with the adjuvant alone were established as controls. After the challenge with $L$. major the course of infection was followed-up for 7 weeks (Fig. 2A in the absence and Fig. 2B in the presence of adjuvant). In the absence of adjuvant no significant differences in the footpad swelling between control and vaccinated groups were observed (Fig. 2C). When mice were immunized with the recombinant proteins combined with the adjuvant, the LmL3 plus CpG-ODN or LmL5 plus CpG-ODN groups showed a decrease in their lesion size compared to the control (CpG-ODN vaccinated mice) (Fig. 2B). Differences were significant from week 5 to week 7 (Fig. 2C). LmS6 plus CpG-ODN vaccinated mice showed a delay in the evolution of $\mathrm{CL}$ until week 5 , but differences were not maintained at the end of the assay (Fig. 2B and C). Mice vaccinated with the proteins without adjuvant had a number of parasites similar to that of the saline control group mice in their DLN and their spleens (Fig. 2D). An approximately 2-log reduction in parasite burden was observed in the DLN cells from mice immunized with LmL3 plus CpG-ODN or LmL5 plus CpG-ODN, relative to the CpG-ODN control group (Fig. 2E). Moreover, spleens from mice immunized with LmL3 plus CpG-ODN or LmL5 plus CpG-ODN were almost free of parasites, contrary to the other vaccinated groups and controls (Fig. 2D and E).

The immune response induced by vaccination with LmL3 and LmL5 was analyzed before challenge. Co-administration of the antigens with the CpG-ODN adjuvant induced a Th1-biased immune response, which was absent in mice immunized with the proteins alone. A significantly higher antigen-specific production of IFN- $\gamma$ was found after in vitro stimulation with the LmL3 $(P=0.0000146$; $P=0.00001468)$ (Fig. 3A) and $\operatorname{LmL5}(P=0.000015 ; P=0.00029)$ (Fig. 3B) proteins beside an IgG2a dominant antibody response against the vaccine antigen (Fig. $3 \mathrm{C}$ and $\mathrm{D}$ ) when compared with saline and CpG-ODN mice group, respectively. Stimulation with SLA
A

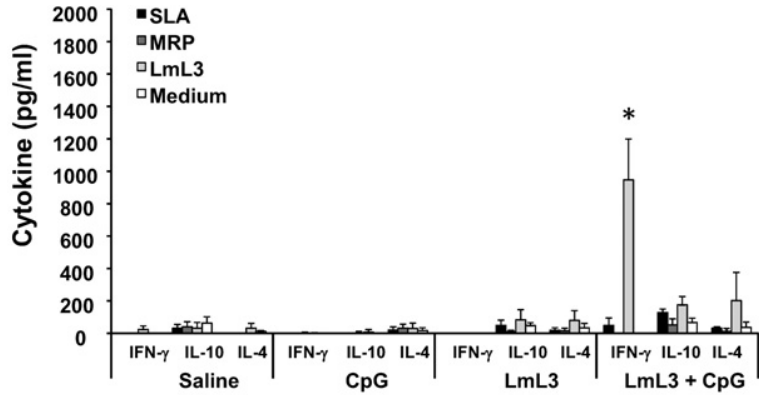

B

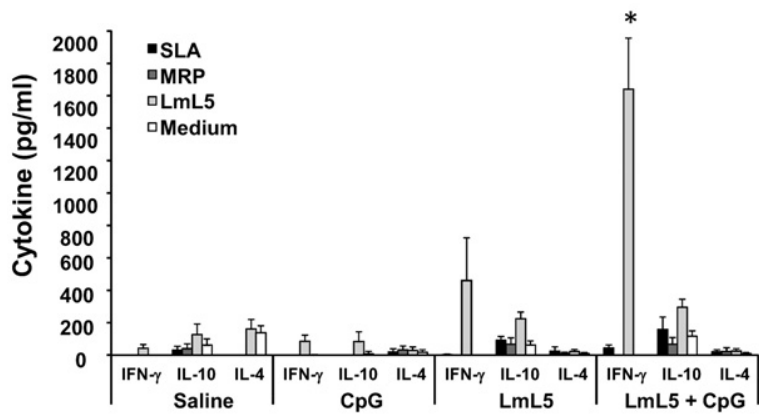

C

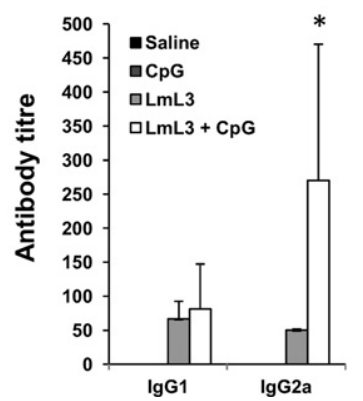

D

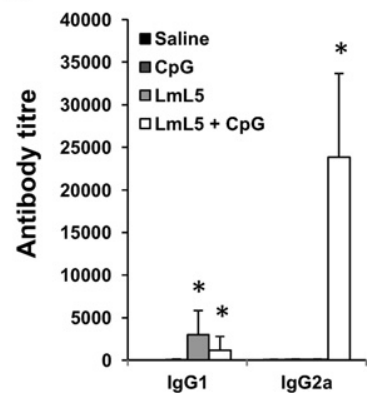

Fig. 3. Immune response elicited in mice vaccinated with $\mathrm{LmL} 3$ and LmL5. Mice ( $n=6$ per group) were vaccinated with $\mathrm{LmL} 3$ in the absence and presence of $\mathrm{CpG}$ ODN (A) or LmL5 alone or plus CPG-ODN (B). Splenocytes from each mouse were independently cultured with medium alone or were in vitro stimulated with soluble Leishmania antigen (SLA), with mouse ribosomal proteins (MRP) and with the corresponding recombinant protein. Splenocytes from control mice, inoculated with saline or CpG-ODN, were stimulated with the same protein preparations in similar conditions. Culture supernatants were collected after $48 \mathrm{~h}$ and the levels of IFN- $\gamma$, IL-10 and IL- 4 were independently measured by sandwich ELISA. Mean + SD are shown. $\left({ }^{*} P<0.05\right.$ indicates a statistically difference between vaccinated mice relative to saline and CpG-ODN controls groups). Anti-LmL3 (C) and anti-LmL5 (D) IgG1 and IgG2a antibody titres were individually determined in the indicated mice groups ( $n=6$ per group) by ELISA. Sera were assayed from $1 / 100$ to $1 / 200,000$ and horseradish peroxidase-conjugated anti-mouse IgG1 (1/1000) or IgG2a (1/500) were used as the secondary antibodies. Mean plus SD are shown. $\left({ }^{*} P<0.05\right.$ significant differences between vaccinated and control mice). Results in each panel are representative of $\geq 2$ independent experiments.

or MRP did not induce cytokine secretion by spleen cells (Fig. 3A and $\mathrm{B})$.

Immune responses were also studied after challenge. Upon in vitro stimulation with the recombinant proteins, $\mathrm{LmL} 3$ plus $\mathrm{CPG}$ ODN (Fig. 4A) or LmL5 plus CpG-ODN (Fig. 4B) vaccinated mice displayed a significant increase in the LmL3 or LmL5 driven IFN$\gamma$ production $(P=0.00009$ and $P=0.0005$, respectively) paralleled by a decrease in IL-10 secretion $(P=0.009$ and $P=0.00001$, respectively), relative to the $\mathrm{CpG}-\mathrm{ODN}$ control group. These results are in accordance with the predominant IgG2a antigen-specific antibody response against LmL3 (Fig. 4C) and LmL5 (Fig. 4D), although 
A

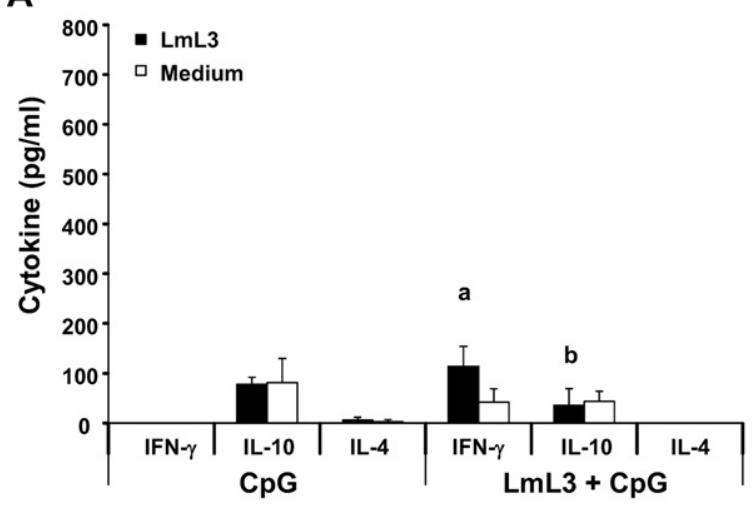

B

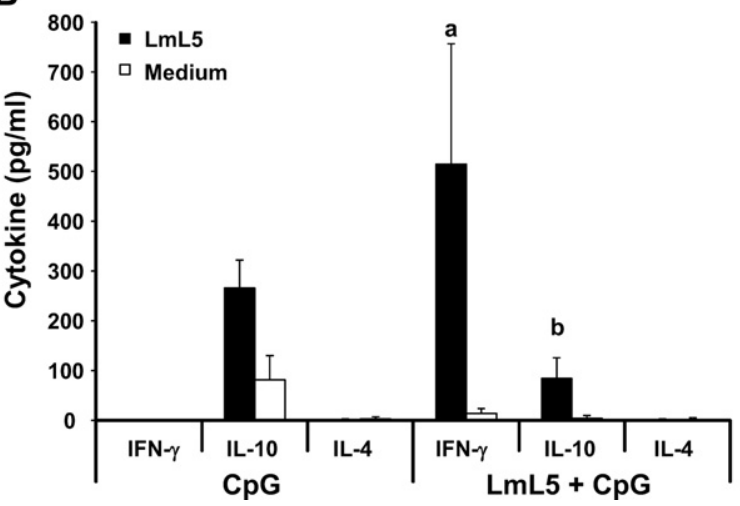

C

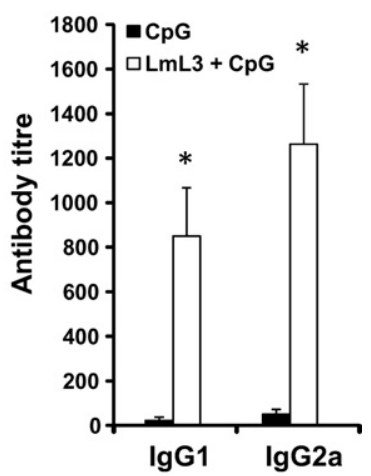

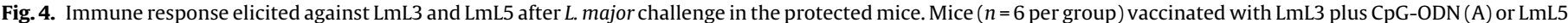

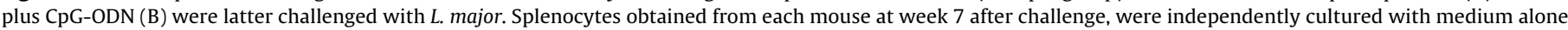

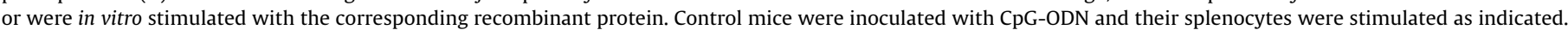

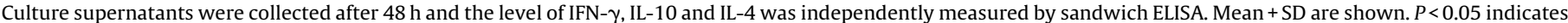

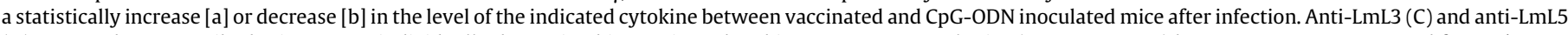

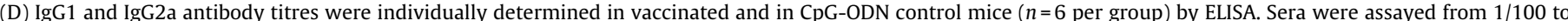

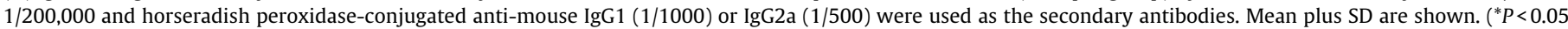
indicates significant differences between vaccinated and CpG-ODN control mice after infection). Results in each panel are representative of 3 independent experiments.

anti-LmL3 and anti-LmL5 IgG1antibodies were also detected (Fig. 4C and D).

The cellular response against SLA was also analyzed in the protected mice after challenge. Secretion of IFN- $\gamma$ was significantly higher in LmL3 plus CpG-ODN or LmL5 plus CpG-ODN vaccinated

A

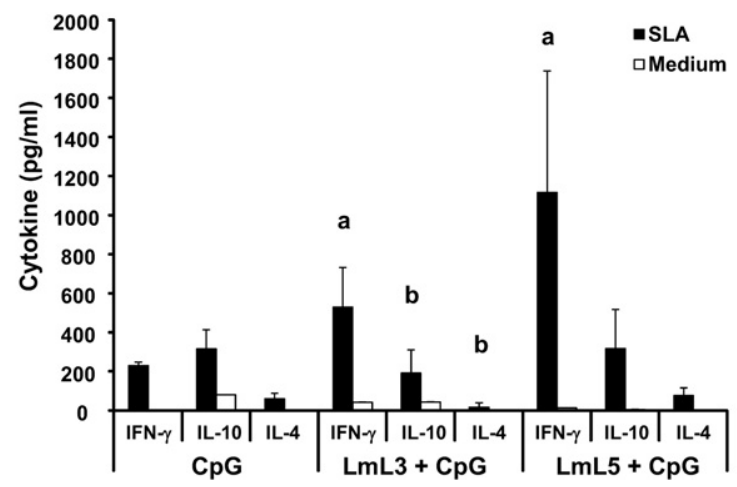

mice, when compared to mice inoculated with CpG-ODN $(P=0.014$ and $P=0.017$, respectively) (Fig. 5A). In LmL3 plus CpG-ODN vaccinated mice a decrease in the SLA-dependent IL-10 production was also observed when compared with $\mathrm{CPG}$-ODN immunized mice $(P=0.009)$. An increment in the SLA-specific IgG2a antibodies was

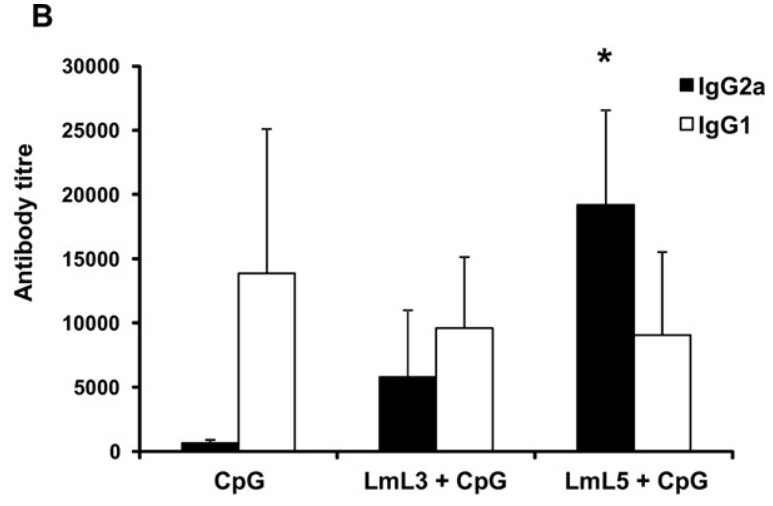

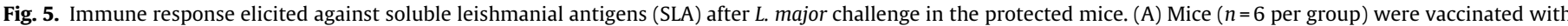

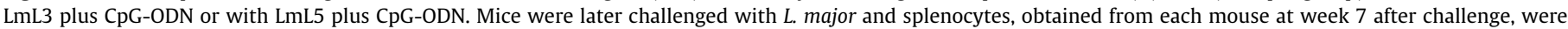

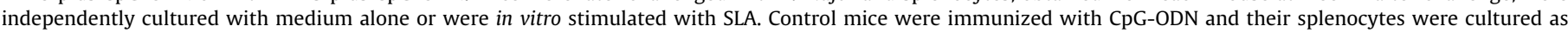

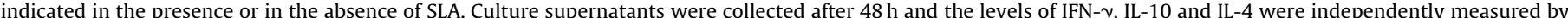

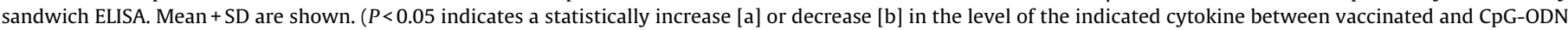

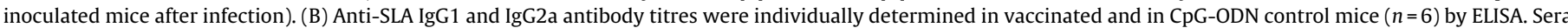

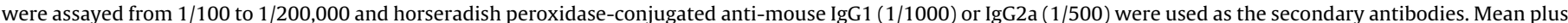

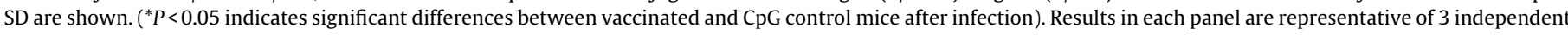
experiments. 
A

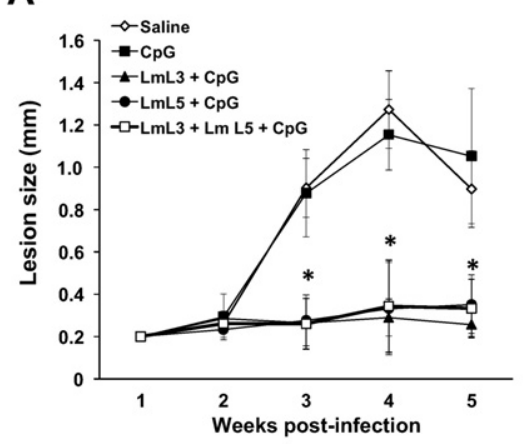

B

\begin{tabular}{|c|c|c|c|}
\hline \multicolumn{4}{|c|}{ Saline } \\
\hline Group $\quad$ Week & $3^{\text {rd }}$ & $4^{\text {th }}$ & $5^{\text {th }}$ \\
\hline $\begin{array}{l}\mathrm{LmL3}+\mathrm{CpG} \\
\mathrm{LmL5}+\mathrm{CpG}\end{array}$ & $\begin{array}{l}8.7 \times 10^{-8} \\
3.7 \times 10^{-9}\end{array}$ & $\begin{array}{l}1.2 \times 10^{-9} \\
6.1 \times 10^{-9}\end{array}$ & $\begin{array}{l}4.5 \times 10^{-7} \\
8.2 \times 10^{-7}\end{array}$ \\
\hline $\mathrm{LmL}_{3}+\mathrm{LmL} 5+\mathrm{CpG}$ & $9.0 \times 10^{-9}$ & $6.2 \times 10^{-10}$ & $5.5 \times 10^{-7}$ \\
\hline \multicolumn{4}{|c|}{ CpG-ODN } \\
\hline Group Week & $3^{\text {rd }}$ & $4^{\text {th }}$ & $5^{\text {th }}$ \\
\hline $\begin{array}{l}\mathrm{LmL3}+\mathrm{CpG} \\
\mathrm{LmL5}+\mathrm{Cpg}\end{array}$ & $\begin{array}{l}1.7 \times 10^{-5} \\
4.3 \times 10^{-6}\end{array}$ & $\begin{array}{l}1.1 \times 10^{-8} \\
5.6 \times 10^{-8}\end{array}$ & $\begin{array}{l}5.8 \times 10^{-5} \\
8.8 \times 10^{-5}\end{array}$ \\
\hline $\mathrm{LmL} 3+\mathrm{LmL} 5+\mathrm{CpG}$ & $9.2 \times 10^{-6}$ & $7.3 \times 10^{-9}$ & $6.8 \times 10^{-5}$ \\
\hline
\end{tabular}

C D
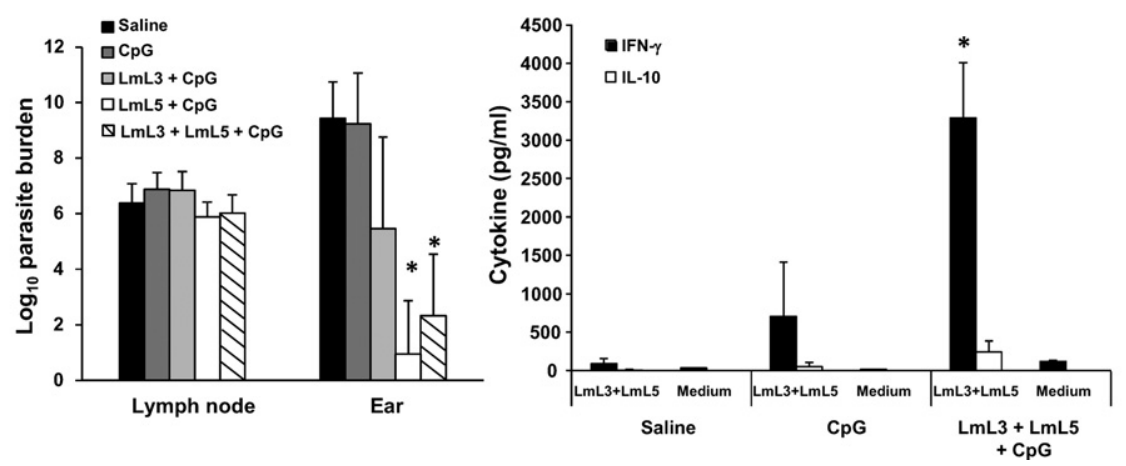

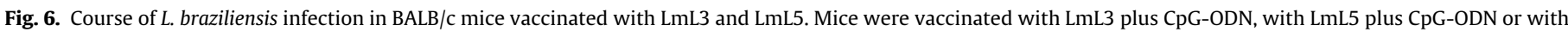

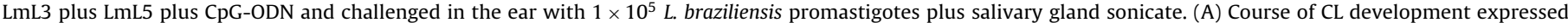

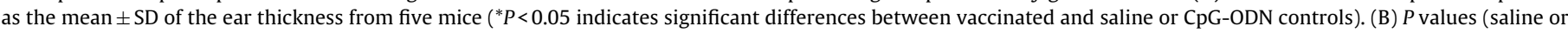

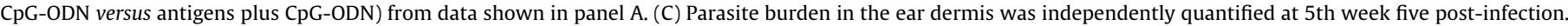

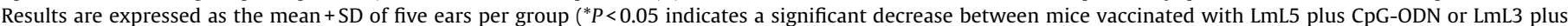

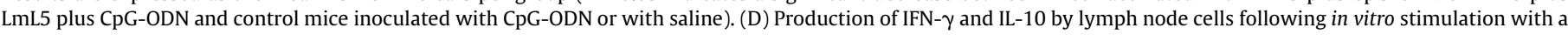

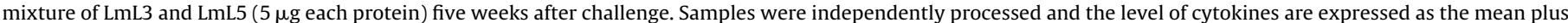

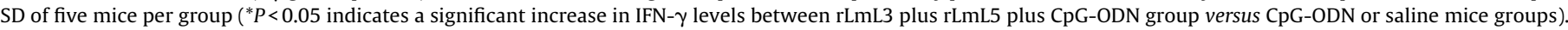
Results in each panel are representative of 2 independent experiments.

observed in LmL3 plus CpG-ODN and LmL5 plus CpG-ODN groups relative to CpG-ODN group, only significant in LmL5 plus CpG-ODN group $(P=0.014)$ (Fig. 5B). The IgG1 response to SLA was similar between the three groups (Fig. 5B).

\subsection{Vaccination with LmL3 plus CpG-ODN and LmL5 plus $C p G-O D N$ protects $B A L B / c$ mice against $L$. braziliensis challenge}

We also investigated whether immunization with LmL3 or LmL5 was able to confer protection in an experimental model of New World CL. Mice were independently vaccinated with LmL3 or LmL5 combined with CpG-ODN or with a mixed formulation of LmL3 and LmL5 plus CpG-ODN. The outcome of infection with L. braziliensis inoculated in the presence of insect vector saliva was evaluated. Ear lesions of vaccinated mice groups were significantly smaller to control groups (saline or CpG-ODN) (Fig. 6A and B). Parasite burden in the ear dermis of the three vaccinated groups was lower than the parasite load of the control groups. Differences with both control groups were significant in the LmL5 plus CpG-ODN $(P=0.00016)$ and in the LmL3 plus LmL5 plus CpG-ODN groups $(P=0.005)$ (Fig. 6C). Similar parasite burden was detected in the DLN of controls and vaccinated mice (Fig. 6C). Finally, to analyze the cellular response elicited against the vaccine antigens, DLN cells from mice immunized with the mixed formulation and both control groups were stimulated with a mixture of the LmL3 and LmL5 recombinant proteins. A significant increase in antigen-specific IFN- $\gamma$ production was observed in the protected mice relative to both control groups $(P=0.015$ for saline and $P=0.007$ for $\mathrm{CpG}-\mathrm{ODN}$ ).

\section{Discussion}

Given that vaccines based on Leishmania ribosomal preparations have induced protection against disease development when immunized with Th1 inducing adjuvants [4-6] we have moved toward the identification of protective antigens in the Leishmania ribosome.

A few ribosome structural proteins have been described as antigenic in canine or human leishmaniasis, including the P0 [16,17], the L6 [18] and, recently, the L25 and L23a proteins [19]. The four ribosomal proteins identified herein (S4, S6, L3 and L5) are antigenic in different forms of the disease caused by distinct parasite species such as $L$. chagasi and L. braziliensis (in humans) and L. infantum (in dogs). Although the recombinant proteins were obtained from L. major DNA the high degree of sequence conservation existing between Leishmania parasite ribosomal orthologues may explain the observed cross-reactivity. Even though it was observed some variability in the recognition between human and canine VL sera (also between VL and MCL human patients) as occur with other parasite antigens [20], our data allow to conclude that the four studied proteins interact with the host immune system, in spite of differences in parasite species and disease forms.

Next, the protective capacities of the characterized antigens were evaluated. Previous reports have identified the prophylactic capacities of three structural proteins of the parasite ribosome: P0 [7], L22 and S19 [21]. Combination of the CpG-ODN adjuvant with the LmL3 or LmL5 proteins induced an immune state that was able to control CL disease due to $L$. major infection in susceptible 
$\mathrm{BALB} / \mathrm{c}$ mice. The immune correlate of protection was the induction of a Th1-like response specific for the recombinant LmL3 or LmL5 proteins. The magnitude of antigen dependent IFN- $\gamma$ secretion and antigen specific IgG2a titers were higher in the LmL5 plus CpG-ODN vaccinated mice than in mice immunized with $\mathrm{LmL} 3$ plus $\mathrm{CPG}-\mathrm{ODN}$ based vaccine. Antigen specific Th1 responses induced upon vaccination were maintained after infection. Vaccines also controlled the LmL3 and LmL5-driven IL-10 responses induced after infection. However, the presence of IgG1 antibodies specific for LmL3, LmL5 is indicating that the Th2 immune response elicited against them by $L$. major infection was not completely abrogated by the vaccines. The Th2-biased response induced after infection against LmL3 and by LmL5 proteins occurring in human or dogs was also observed in mice vaccinated with the proteins without the adjuvant, since in these mice antibodies elicited against both antigens were of the IgG1 isotype (data not shown). This fact points out the importance of the adjuvant in ribosome-based vaccines. Thus, CpG-ODN motifs were able to redirect toward a protective Th1-like profile the response against LmL3 and LmL5 as also occur with other vaccine formulations like these based on LmSTI1 plus CpG-ODN soluble and particulate preparations [22].

Although the protective effects of the LmL3 or the LmL5 plus CpG-ODN documented here were analyzed in a model that employ a syringe-based challenge the results obtained allowed us to conclude that the protective effect of the immunization of LmL3 and LmL5 based vaccines is similar to that obtained with various parasite proteins assayed for protection under similar conditions [23].

Cross-prophylactic properties of the LmL3 or LmL5 based vaccines were also tested in an experimental model of CL caused by $L$. braziliensis [24]. Many of the proteins known to induce protection against $L$. major or $L$. infantum infection in BALB/C mice, were not able to control the CL caused by $L$. braziliensis $[25,26]$ or only induce partial protection [27]. In this model, BALB/c mice show lesions in the challenge site (ear) that are resolved after induction of a Th1 type immune response that eliminates parasites from ears, maintaining a chronic infection in the DLN [24]. Since co-inoculation of vector saliva and $L$. braziliensis led to a significant exacerbation of both lesion size and parasites load in the mice experimental model [28] we have employed here a syringe-based challenge in which vector saliva and stationary parasites are co-inoculated. Interestingly, we found that ear inflammatory lesions were almost absent in vaccinated mice and a very low number of parasites was detected in the ears 5 weeks after challenge, especially in mice vaccinated with LmL5 plus CpG-ODN or with a combination of both ribosomal proteins and $\mathrm{CpG}-\mathrm{ODN}$. Given the antigen specific IFN- $\gamma$ mediated response was observed in protected mice, it can be suggested that IFN- $\gamma$-secreting cells may have migrated to the infected ear early after challenge, promoting parasite killing in the absence of an inflammatory response of a high magnitude. These cells, however, are unable to destroy parasites in the DLNs as also occur in the infected controls in accordance to what it has been previously reported for this experimental model of infection [24]. Since results obtained by our group have shown that immunization with LmL3 or LmL5 ribosomal antigens combined with CpG-ODN also reduced parasite loads in BALB/c mice infected with $L$. chagasi (manuscript in preparation), we conclude that the LML3 and LmL5 antigens, formulated with Th1 inducing adjuvants should be considered in the development of vaccines against leishmaniasis.

\section{Conclusions}

In this work four new antigenic proteins have been described in Leishmania ribosome: S4, S6, L3 and L5. Recombinant proteins obtained from $L$. major were recognized by the sera from individuals infected with different parasite species and suffering different forms of the disease. Two of them, LmL3 or LmL5 were able to protect mice against CL caused by $L$. major and by $L$. braziliensis when administered in the presence of a Th1 inducing adjuvant. In both models, protection was associated with the induction of antigen-specific IFN- $\gamma$ mediated responses, but also with control of the antigen dependent production of IL-10 in some cases. Altogether, data presented here are indicating that LmL3 and LmL5 may be considered relevant antigens in the formulation of vaccines against leishmaniasis.

\section{Acknowledgments}

We thank Dr Manoel Barral-Netto for critically discussing the project. We thank Libertad Teresa and María Vega for her technical support. We thank Dr Julian de la Horra and Dr. José M Requena for critically discussing the manuscript during the revision process. The study was supported in Spain by grants from Laboratorios LETI S.L.u, from Ministerio de Ciencia e Innovación FIS/PI080101 and FIS PI11/00095 and from the Instituto de Salud Carlos III within the Network of Tropical Diseases Research (RICET RD06/0021/0008). This work was also partially supported by grants from FAPEMIG (CBB-APQ-00496-11), CNPq (APQ-472090/2011-9) and INCT NANO-BIOFAR. EAFC is a grant recipient of CNPq. A CBMSO institutional grant from Fundación Ramón Areces is also acknowledged.

\section{Appendix A. Supplementary data}

Supplementary data associated with this article can be found, in the online version, at http://dx.doi.org/10.1016/j.vaccine. 2012.12.071.

\section{References}

[1] Herwaldt BL. Leishmaniasis. Lancet 1999;354(9185):1191-9.

[2] Kaye P, Scott P. Leishmaniasis: complexity at the host-pathogen interface. Nat Rev Microbiol 2011;9(8):604-15.

[3] Nylen S, Gautam S. Immunological perspectives of leishmaniasis. J Glob Infect Dis 2010;2(2):135-46

[4] Iborra S, Parody N, Abanades DR, Bonay P, Prates D, Novais FO, et al. Vaccination with the Leishmania major ribosomal proteins plus $\mathrm{CpG}$ oligodeoxynucleotides induces protection against experimental cutaneous leishmaniasis in mice. Microbes Infect 2008;10(10-11):1133-41.

[5] Ramirez L, Iborra S, Cortes J, Bonay P, Alonso C, Barral-Netto M, et al. BALB/c mice vaccinated with Leishmania major ribosomal proteins extracts combined with CPG oligodeoxynucleotides become resistant to disease caused by a secondary parasite challenge. J Biomed Biotechnol 2010;2010:181690.

[6] Chavez-Fumagalli MA, Costa MA, Oliveira DM, Ramirez L, Costa LE, Duarte MC et al. Vaccination with the Leishmania infantum ribosomal proteins induces protection in BALB/c mice against Leishmania chagasi and Leishmania amazonensis challenge. Microbes Infect 2010;12(12-13):967-77.

[7] Iborra S, Carrion J, Anderson C, Alonso C, Sacks D, Soto M. Vaccination with the Leishmania infantum acidic ribosomal P0 protein plus CPG oligodeoxynucleotides induces protection against cutaneous leishmaniasis in C57BL/6 mice but does not prevent progressive disease in BALB/c mice. Infect Immun 2005;73(9):5842-52.

[8] Rhee EG, Mendez S, Shah JA, Wu CY, Kirman JR, Turon TN, et al. Vaccination with heat-killed Leishmania antigen or recombinant leishmanial protein and $\mathrm{CpG}$ oligodeoxynucleotides induces long-term memory CD4+ and CD8+ T cell responses and protection against Leishmania major infection. J Exp Med 2002;195(12):1565-73.

[9] Mager WH, Planta RJ, Ballesta JG, Lee JC, Mizuta K, Suzuki K, et al. A new nomenclature for the cytoplasmic ribosomal proteins of Saccharomyces cerevisiae. Nucleic Acids Res 1997;25(24):4872-5.

[10] Shi PY, Maizels N, Weiner AM. Recovery of soluble, active recombinant protein from inclusion bodies. BioTech 1997;23(6):1036-8.

[11] Coelho EA, Ramirez L, Costa MA, Coelho VT, Martins VT, Chavez-Fumagalli MA, et al. Specific serodiagnosis of canine visceral leishmaniasis using Leishmania species ribosomal protein extracts. Clin Vaccine Immunol 2009;16(12):1774-80

[12] Soto M, Requena JM, Quijada L, Perez MJ, Nieto CG, Guzman F, et al. Antigenicity of the Leishmania infantum histones $\mathrm{H} 2 \mathrm{~B}$ and $\mathrm{H} 4$ during canine viscerocutaneous leishmaniasis. Clin Exp Immunol 1999;115(2):342-9.

[13] Soto M, Requena JM, Quijada L, Angel SO, Gomez LC, Guzman F, et al During active viscerocutaneous leishmaniasis the anti-P2 humoral response 
is specifically triggered by the parasite $\mathrm{P}$ proteins. Clin Exp Immunol 1995; 100(2):246-52.

[14] de Moura TR, Oliveira F, Rodrigues GC, Carneiro MW, Fukutani KF, Novais FO, et al. Immunity to Lutzomyia intermedia saliva modulates the inflammatory environment induced by Leishmania braziliensis. PLoS Negl Trop Dis 2010;4(6):e712.

[15] Buffet PA, Sulahian A, Garin YJ, Nassar N, Derouin F. Culture microtitration: a sensitive method for quantifying Leishmania infantum in tissues of infected mice. Antimicrob Agents Chemoter 1995;39(9):2167-8.

[16] Skeiky YA, Benson DR, Elwasila M, Badaro R, Burns Jr JM, Reed SG. Antigens shared by Leishmania species and Trypanosoma cruzi: immunological comparison of the acidic ribosomal P0 proteins. Infect Immun 1994;62(5):1643-51.

[17] Soto M, Requena JM, Quijada L, Guzman F, Patarroyo ME, Alonso C. Identification of the Leishmania infantum $\mathrm{P0}$ ribosomal protein epitope in canine visceral leishmaniasis. Immunol Lett 1995;48(1):23-8.

[18] Thomas MC, Martinez-Carretero E, Carmelo E, Gonzalez AC, Valladares B. Molecular characterization of the Leishmania braziliensis L6 ribosomal protein. J Parasitol 2004;90(4):908-13.

[19] Coelho VT, Oliveira JS, Valadares DG, Chavez-Fumagalli MA, Duarte MC, Lage $\mathrm{PS}$, et al. Identification of proteins in promastigote and amastigote-like Leishmania using an immunoproteomic approach. PLoS Negl Trop Dis 2012;6(1): e1430.

[20] Goto Y, Howard RF, Bhatia A, Trigo J, Nakatani M, Netto EM, et al. Distinct antigen recognition pattern during zoonotic visceral leishmaniasis in humans and dogs. Vet Parasitol 2009;160(3-4):215-20.

[21] Stober CB, Lange UG, Roberts MT, Gilmartin B, Francis R, Almeida R, et al. From genome to vaccines for leishmaniasis: screening 100 novel vaccine candidates against murine Leishmania major infection. Vaccine 2006;24(14): 2602-16.

[22] Badiee A, Jaafari MR, Samiei A, Soroush D, Khamesipour A. Coencapsulation of CpG oligodeoxynucleotides with recombinant Leishmania major stress-inducible protein 1 in liposome enhances immune response and protection against leishmaniasis in immunized BALB/c mice. Clin Vaccine Immunol 2008;15(4):668-74.

[23] Soto M, Ramírez L, Pineda MA, González VM, Entringer PF, Indiani de Oliveira, et al. Searching genes encoding Leishmania antigens for diagnosis and protection. Sch Res Exch 2009;2009:ID173039.

[24] de Moura TR, Novais FO, Oliveira F, Clarencio J, Noronha A, Barral A, et al. Toward a novel experimental model of infection to study American cutaneous leishmaniasis caused by Leishmania braziliensis. Infect Immun 2005;73(9):5827-34.

[25] Salay G, Dorta ML, Santos NM, Mortara RA, Brodskyn C, Oliveira CI, et al. Testing of four Leishmania vaccine candidates in a mouse model of infection with Leishmania (Viannia) braziliensis, the main causative agent of cutaneous leishmaniasis in the New World. Clin Vaccine Immunol 2007;14(9):1173-81.

[26] Tonui WK, Titus RG. Cross-protection against Leishmania donovani but not $L$. braziliensis caused by vaccination with $L$. major soluble promastigote exogenous antigens in BALB/c mice. Am J Trop Med Hyg 2007;76(3):579-84.

[27] Santos DM, Carneiro MW, de Moura TR, Fukutani K, Clarencio J, Soto M, et al. Towards development of novel immunization strategies against leishmaniasis using PLGA nanoparticles loaded with kinetoplastid membrane protein-11. Int J Nanomedicine 2012;7:2115-27.

[28] Samuelson J, Lerner E, Tesh R, Titus R. A mouse model of Leishmania braziliensis braziliensis infection produced by coinjection with sand fly saliva. J Exp Med 1991;173(1):49-54. 